\title{
Applicability of the Wigner functional approach to evolution of quantum fields
}

\author{
Andrey Leonidov ${ }^{1,2,3, \star}$ and Anna Radovskaya ${ }^{1, \star \star}$ \\ ${ }^{1}$ P.N. Lebedev Physics Institute of the Russian Academy of Sciences, Moscow, Russia \\ ${ }^{2}$ Moscow Engineering Physics Institute, Moscow, Russia \\ ${ }^{3}$ Moscow Institute of Physics and Technology, Moscow, Russia
}

\begin{abstract}
Evolution of highly excited quantum field is considered in the framework of Keldysh formalism . It is demonstrated that leading order (LO) term of semiclassical approximation appears as well-known Classical Statistical Approximation (CSA). In simple case of spatially homogeneous scalar field analytical expressions for leading and next-toleading (NLO) order are presented. It is shown that the range of applicability of CSA strongly depends on the properties of the initial state of the system.
\end{abstract}

\section{Introduction}

The problem of description of the evolution of highly excited quantum has recently attracted considerable attention of theorists working on problems in various domains of theoretical physics. The relevant examples include, in particular, studies of initial stages of ultrarelativistic heavy ion collisions $[1,8]$, heating of the Universe after inflation $[9,11]$, particle production in ultrastrong fields $[12,15]$, thermalization in ultracold quantum gases $[16,17]$. All the above-mentioned systems share the basic common feature - their initial state can not be described as the vacuum one. This means that the standard S-matrix formalism is not applicable here. In fact, for highly excited fields it is necessary to give a correct specification of the initial state and be able to describe its subsequent temporal evolution. The Keldysh technique $[18,20]$ provides all the necessary technical tools for solving such problems.

In the previous work [21] we demonstrated that the Keldysh formalism applied to description of evolution of excited fields results in its Leading Order in so-called Classical Statistical Approximation (CSA). CSA is based on the fact that summation of leading quantum corrections can be cast in the form of integration over initial conditions for classical trajectories with the weight given by the Wigner functional. It is interesting to note that similar approach was used in atomic and chemical physics [22],[23].

In this paper we analyze the applicability of the Wigner functional approach to evolution of highly excited quantum fields by calculatingf the Next-to-Leading corrections to several observables. We show that this applicability does directly depend on the properties of the initial state of the system and find out range of the validity of the Wigner functional approach considering homogeneous scalar field model as an example.

\footnotetext{
$\star^{\star}$ e-mail: leonidov@lpi.ru

$\star \star$ e-mail: raan@1pi.ru
} 


\section{General formalism}

In this section we will shortly remind general formalism for calculation of observables within the Keldysh technique suitable to our problem. Out of equilibrium an expectation value of observable $F(\hat{\varphi})$ at the moment $t_{1}$ can be calculated as a trace with density matrix as

$$
\langle F(\hat{\varphi})\rangle_{t_{1}}=\operatorname{tr}\left(F(\hat{\varphi}) \hat{\rho}\left(t_{1}\right)\right)=\int \mathfrak{D} \xi(\vec{x}) F(\xi)\left\langle\xi\left|\hat{U}\left(t_{1}, t_{0}\right) \hat{\rho}\left(t_{0}\right) \hat{U}\left(t_{0}, t_{1}\right)\right| \xi\right\rangle
$$

where evolution of the density matrix $\hat{\rho}(t)$ is governed by the evolution operator $\hat{U}\left(t, t_{0}\right)$

$$
\hat{\rho}(t)=\hat{U}\left(t, t_{0}\right) \hat{\rho}\left(t_{0}\right) \hat{U}\left(t_{0}, t\right),
$$

and $|\xi\rangle$ is an eigenstate of the field operator $\hat{\varphi}(\vec{x})|\xi\rangle=\xi(\vec{x})|\xi\rangle$ and $\int \mathfrak{D} \xi(\vec{x})$ is a path integral over all possible 3-d functions originating from unity operator $\hat{1}=\int \mathfrak{D} \xi(\vec{x})|\xi\rangle\langle\xi|$.

The matrix elements of the evolution operator can be represented as the path integrals over 4-d functions $\mathcal{D} \eta(t, \vec{x})$

$$
\begin{aligned}
& \left\langle\xi\left|\hat{U}\left(t_{1}, t_{0}\right)\right| \xi_{1}\right\rangle=\int_{\eta_{F}\left(t_{0}, \vec{x}\right)=\xi_{1}(\vec{x})}^{\eta_{B}\left(t_{1}, \vec{x}\right)=\xi(\vec{x})} \mathcal{D} \eta_{F}(t, \vec{x}) e^{i S\left[\eta_{F}\right]}, \\
& \left\langle\xi_{2}\left|\hat{U}\left(t_{0}, t_{1}\right)\right| \xi\right\rangle=\int_{\eta_{B}\left(t_{0}, \vec{x}\right)=\xi_{2}(\vec{x})}^{\left.\eta^{\prime}\right)=\xi(\vec{x})} \mathcal{D} \eta_{B}(t, \vec{x}) e^{-i S\left[\eta_{B}\right]}
\end{aligned}
$$

Here $\eta_{F}\left(t_{1}, \vec{x}\right)$ and $\eta_{F}\left(t_{1}, \vec{x}\right)$ are the field that lies on the forward $\left(\eta_{F}\right)$ and backward $\left(\eta_{B}\right)$ sides of Keldysh contour (see [21] for details). Combining all together we obtain

$$
\begin{gathered}
\langle F(\hat{\varphi})\rangle_{t_{1}}=\int \mathcal{D} \xi \int \mathcal{D} \xi_{1} \int \mathcal{D} \xi_{2}\left\langle\xi_{1}\left|\hat{\rho}\left(t_{0}\right)\right| \xi_{2}\right\rangle \times \\
F(\xi) \int_{\eta_{F}\left(t_{0}, \vec{x}\right)=\xi_{1}(\vec{x})}^{\eta_{F}\left(t_{1}, \vec{x}\right)=\xi(\vec{x})} \mathcal{D} \eta_{F}(t, \vec{x}) \int_{\eta_{B}\left(t_{0}, \vec{x}\right)=\xi_{2}(\vec{x})}^{\eta_{B}\left(t_{1}, \vec{x}\right)=\xi(\vec{x})} \mathcal{D} \eta_{B}(t, \vec{x}) e^{i S\left[\eta_{F}\right]-i S\left[\eta_{B}\right]} .
\end{gathered}
$$

The next step is to perform technical trick and extend Keldesh contour to infinity. After that we can introduce new field as

$$
\phi_{c}=\frac{\eta_{F}+\eta_{B}}{2}, \quad \phi_{q}=\eta_{F}-\eta_{B}
$$

and define the Keldysh action $S_{K}\left[\eta_{F}, \eta_{B}\right]=S\left[\eta_{F}\right]-S\left[\eta_{B}\right]$ in order to obtain general expression

$$
\begin{gathered}
\langle F(\hat{\varphi})\rangle_{t_{1}}=\int \mathcal{D} \chi_{1} \int \mathcal{D} \xi_{1} \int \mathcal{D} \xi_{2}\left\langle\xi_{1}\left|\hat{\rho}\left(t_{0}\right)\right| \xi_{2}\right\rangle \times \\
\int_{\phi_{c}\left(t_{0}, \vec{x}\right)=\frac{\xi_{1}(\vec{x})+\xi_{2}(\vec{x})}{2}} \mathcal{D} \phi_{c} \int_{\phi_{q}\left(t_{0}, \vec{x}\right)=\chi_{1}(\vec{x})-\xi_{1}(\vec{x})=0} \mathcal{D} \phi_{q} F\left(\phi_{c}\left(t_{1}\right)\right) e^{i S_{K}\left[\phi_{c}, \phi_{q}\right]} .
\end{gathered}
$$

Eq.(5) represents our main tool for calculation of observables however we need to specify Lagrangian in order to calculate certain results. 


\section{Scalar $\varphi^{4}$ theory}

The simplest model which has necessary nontrivial dynamical behavior is a scalar $\varphi^{4}$ model with quartic interaction term.

$$
\begin{gathered}
\mathcal{L}=\frac{1}{2} \partial_{\mu} \varphi \partial^{\mu} \varphi-\frac{g^{2}}{4} \varphi^{4}+J \varphi, \\
S=\int d^{4} x \mathcal{L} .
\end{gathered}
$$

Here $J(t, \vec{x})$ is an auxiliary source which we keep in order to perform semiclassical decomposition.

For the Lagrangian (6) the Keldysh actions reads

$$
\begin{gathered}
S_{K}\left[\phi_{c}, \phi_{q}\right]=\int d^{3} x \dot{\phi}_{c}\left(t_{0}, \vec{x}\right)\left(\xi_{1}(\vec{x})-\xi_{2}(\vec{x})\right)-\int d^{3} x \int_{t_{0}}^{\infty} d t\left(\phi_{q} A\left[\phi_{c}\right]-\frac{g^{2}}{4} \phi_{c} \phi_{q}^{3}\right), \\
A\left[\phi_{c}\right]=\left[\ddot{\phi}_{c}+g^{2} \phi_{c}^{3}-J\right] .
\end{gathered}
$$

Here term $\phi_{q}\left(t_{0}, \vec{x}\right)=\xi_{1}(\vec{x})-\xi_{2}(\vec{x})$ appears from integration by parts. Note that $A\left[\phi_{c}\right]=0$ corresponds to projecting onto the classical equation of motion for the Lagrangian Eq.(6). It is important that the source $J$ connected to both fields on upper and lower sides of Keldysh contour in similar way so we get term $J\left(\eta_{F}-\eta_{B}\right)=J \phi_{q}$.

It is clear that path integrals of (5) with this action can not be calculated explicitly. The systematic procedure we employ is expansion in $\phi_{q}$ in (7) around its saddle-point value. This expansion is, in fact, a semiclassical one. This can be seen by restoring $\hbar$ in the action and replacing $\phi_{q} \rightarrow \hbar \phi_{q}$ so the only remaining dependence on $\hbar$ is in $\phi_{q}^{3}$ which is proportional to $\hbar^{2}$ and

$$
e^{-i \frac{g^{2} \hbar^{2}}{4} \int_{t_{0}}^{\infty} d t \int d^{3} x \phi_{c} \phi_{q}^{3}}=\underbrace{1}_{L O}-\underbrace{\frac{i g^{2} \hbar^{2}}{4} \int_{t_{0}}^{\infty} d t \int d^{3} x \phi_{c} \phi_{q}^{3}}_{N L O}+\ldots
$$

This expansion shows that the LO contribution contains quantum fluctuation up to one loop order.

\subsection{Leading Order}

The Leading Order (LO) contribution to observables corresponds to the first term in decomposition (8). In this case we are able to perform functional integration over field $\phi_{q}$ resulting with the functional delta-function which project $\phi_{c}$ onto the solution of classical equation of motion of the Lagrangian (6). As the next step we insert the "initial velocity" as $1=\int \mathfrak{D} p(\vec{x}) \delta\left(\tilde{p}(\vec{x})-\dot{\phi}_{c}\left(t_{0}, \vec{x}\right)\right)$ in order to integrate over $\phi_{c}$ (see [21] for details). After substitution $\alpha=\frac{\xi_{1}+\xi_{2}}{2}, \beta=\xi_{1}-\xi_{2}$ we end up with

$$
\left.\langle F(\hat{\varphi})\rangle_{t_{1}}=\int \mathfrak{D} \alpha(\vec{x}) \mathfrak{D} p(\vec{x}) f_{W}[\alpha(\vec{x}), p(\vec{x})), t_{0}\right] F\left(\phi_{c l}\left(t_{1}, \vec{x}\right)\right),
$$

where the Wigner functional is commonly defined as

$$
f_{W}\left[\alpha(\vec{x}), p(\vec{x}), t_{0}\right]=\int \mathfrak{D} \beta(\vec{x})\left\langle\alpha+\frac{\beta}{2}\left|\hat{\rho}\left(t_{0}\right)\right| \alpha-\frac{\beta}{2}\right\rangle \times \exp \left(i \int d^{3} x p(\vec{x}) \beta(\vec{x})\right) .
$$


Here $\phi_{c l}$ is the solution of classical equation of motion

$$
\partial_{\mu} \partial^{\mu} \phi_{c l}+g^{2} \phi_{c l}^{3}=0
$$

with initial conditions given by

$$
\phi_{c l}\left(t_{0}, \vec{x}\right)=\alpha(\vec{x}), \quad \dot{\phi}_{c l}\left(t_{0}, \vec{x}\right)=p(\vec{x})
$$

and at zero axillary source $J(t, \vec{x})$.

Expression (9) was earlier derived by other methods in [1, 4], see also [19],[24] . The general recipe for calculation at the LO level is the following: in order to obtain expectation value of observable at a given time moment $t_{1}$ one need to find solution of classical equation of motion as a function of the field and its time derivative at the initial time and average over initial conditions with weight of Wigner functional. Note that choice of Wigner functional (or density matrix at initial time) lies outside the method itself and depends on problem that should be solved. For certain choice of Wigner functional the expression (9) represent the Classical Statistical Approximation widely used in the context of ultrarelativistic heavy ion collisions.

Let us introduce new notation for averaging over initial conditions

$$
\left.\langle O\rangle_{i . c .}=\int \mathfrak{D} \alpha(\vec{x}) \mathfrak{D} p(\vec{x}) f_{W}[\alpha(\vec{x}), p(\vec{x})), t_{0}\right] \mathcal{O} .
$$

Then we can rewrite (9) simply as

$$
\langle F(\hat{\varphi})\rangle_{t_{1}}^{L O}=\left\langle F\left(\phi_{c l}\left(t_{1}, \vec{x}\right)\right)\right\rangle_{i . c .} .
$$

\subsection{Next-to-Leading Order}

In order to decide if expansion over $\phi_{q}$ is valid we should consider Next-to-Leading Order (NLO) term in (8). With this $\phi_{q}^{3}$ part the path integration over $\phi_{q}$ can not be done as easy as at LO level. However one can note that each $\phi_{q}$ can be replaced by functional derivative over source $J$ due to $\phi_{q} J$ term in the Keldysh action (7)

$$
\frac{\delta}{\delta J(t, \vec{x})} e^{i S_{K}\left[\phi_{c}, \phi_{q}\right]}=-i \phi_{q}(t, \vec{x}) e^{i S_{K}\left[\phi_{c}, \phi_{q}\right]} .
$$

After that we can perform functional integration over $\phi_{q}$ and $\phi_{c}$ in order to obtain answer for expectation value of the observable up to NLO level

$$
\langle F(\hat{\varphi})\rangle_{t_{1}}^{L O+N L O}=\left\langle F\left(\phi_{c l}\left(t_{1}, \vec{x}\right)\right)+\left.\frac{g^{2}}{4} \int_{t_{0}}^{t_{1}} d t_{2} \int d^{3} x_{2} \phi_{c l}\left(t_{2}, \vec{x}_{2}\right) \frac{\delta^{3} F\left(\phi_{c l}\left(t_{1}, \vec{x}\right)\right)}{\delta J^{3}\left(t_{2}, \vec{x}_{2}\right)}\right|_{J=0}\right\rangle_{i . c .} .
$$

The expression above shows that there is no necessity in any new information for evaluation of NLO correction. We should find classical trajectory as a function of initial conditions, perform three variations over auxiliary source, integrate over intermediate time and average with the Wigner functional. These calculations seem to be rather tedious however there is a technical trick.

Let us define $k$-th variation of the classical solution over source $J$ as

$$
\frac{\delta^{k} \phi_{c l}\left(t_{1}, \vec{x}_{1}\right)}{\delta J^{k}\left(t_{2}, \vec{x}_{2}\right)}=\Phi_{k}\left(t_{1}, \vec{x}_{1} ; t_{2}, \vec{x}_{2}\right)
$$


Then

$$
\begin{aligned}
\frac{\delta^{3} F\left(\phi_{c l}\left(t_{1}, \vec{x}_{1}\right)\right)}{\delta J^{3}}=\frac{\partial F}{\partial \phi_{c l}} \Phi_{3}\left(t_{1}, \vec{x}_{1} ; t_{2}, \vec{x}_{2}\right)+3 \frac{\partial^{2} F}{\partial \phi_{c l}^{2}} \Phi_{1}\left(t_{1}, \vec{x}_{1} ; t_{2}, \vec{x}_{2}\right) \Phi_{2}\left(t_{1}, \vec{x}_{1} ; t_{2}, \vec{x}_{2}\right) & \\
& +\frac{\partial^{3} F}{\partial \phi_{c l}^{3}} \Phi_{1}^{3}\left(t_{1}, \vec{x}_{1} ; t_{2}, \vec{x}_{2}\right) .
\end{aligned}
$$

Variations $\Phi_{k}\left(t_{1}, \vec{x}_{1} ; t_{2}, \vec{x}_{2}\right)$ can be found by variation of the classical EoM

$$
\frac{\delta^{3}}{\delta J^{3}\left(t_{2}, \vec{x}_{2}\right)}\left(\partial_{\mu} \partial^{\mu} \phi_{c l}\left(t_{1}, \vec{x}_{1}\right)+g^{2} \phi_{c l}^{3}\left(t_{1}, \vec{x}_{1}\right)=J\left(t_{1}, \vec{x}_{1}\right)\right)
$$

that gives

$$
\begin{aligned}
L_{t_{1}} \Phi_{1}\left(t_{1}, \vec{x}_{1} ; t_{2}, \vec{x}_{2}\right) & =\delta\left(t_{1}-t_{2}\right) \delta^{(3)}\left(\vec{x}_{1}-\vec{x}_{2}\right) \\
L_{t_{1}} \Phi_{2}\left(t_{1}, \vec{x}_{1} ; t_{2}, \vec{x}_{2}\right) & =-6 g^{2} \phi_{c l}\left(t_{1}, \vec{x}_{1}\right) \Phi_{1}^{2}\left(t_{1}, \vec{x}_{1} ; t_{2}, \vec{x}_{2}\right) \\
L_{t_{1}} \Phi_{3}\left(t_{1}, \vec{x}_{1} ; t_{2}, \vec{x}_{2}\right) & =-6 g^{2} \Phi_{1}^{3}\left(t_{1}, \vec{x}_{1} ; t_{2}, \vec{x}_{2}\right)-18 g^{2} \phi_{c l}\left(t_{1}, \vec{x}_{1}\right) \Phi_{1}\left(t_{1}, \vec{x}_{1} ; t_{2}, \vec{x}_{2}\right) \Phi_{2}\left(t_{1}, \vec{x}_{1} ; t_{2}, \vec{x}_{2}\right) \\
L_{t_{1}} & =\partial_{t_{1}}^{2}-\partial_{\vec{x}_{1}}^{2}+3 g^{2} \phi_{c l}^{2}\left(t_{1}, \vec{x}_{1}\right) .
\end{aligned}
$$

With help of differential equations (18) we are able to calculate NLO correction to any observable without knowledge of exact dependence of the classical solution $\phi_{c l}$ on auxiliary source $J(t, \vec{x})$. Although the recipe for NLO calculation is fulfilled we can not use it directly with Lagrangian (6). Analytical solution of equation of motion even for zero source can not be found. In the next section we consider simplification of described model that allow

\section{Spatially homogenous analytical solution}

Let us consider $\varphi^{4}$ toy model . We suppose that all spatial gradients small enough to be neglected. Although this assumption takes us away from implementation to real physical systems it let us perform a lot of work analytically and reveal general features that can be extended to full theory.

In spatially homogeneous case $\partial_{i} \varphi(t, \mathbf{x})=0$, than

$$
\begin{gathered}
S=V_{3} \int d t\left(\frac{1}{2} \dot{\varphi}^{2}-\frac{g^{2}}{4} \varphi^{4}+J \varphi\right), \\
V_{3}=\int d^{3} x .
\end{gathered}
$$

The equation of motion is

$$
\ddot{\varphi}+g^{2} \varphi^{3}=J .
$$

We can find classical solution of EoM (20) for $\mathrm{J}=0$ in terms of the Jacobi elliptical function $c n$ with module $k^{2}=\frac{1}{2}$ as

$$
\phi_{c l}(t)=\phi_{m} c n\left(\frac{1}{2}, g \phi_{m} t+C\right) \text {. }
$$

The period of this function is $T_{c l}=\frac{4}{g \phi_{m}} K(1 / 2)$, where $\mathrm{K}(1 / 2)$ is the complete elliptic integral of the first kind. Here $\phi_{m}$ and $C$ are the amplitude and the phase of the solution. 
First of all we calculate the field expectation value $\langle\hat{\varphi}\rangle_{t_{1}}$ as a simplest example. In our spatially homogeneous case the eq.(3.2) has the form of

$$
\langle\hat{\varphi}\rangle_{t_{1}}^{L O+N L O}=\left\langle\phi_{c l}\left(t_{1}\right)+\frac{g^{2} V_{3}}{4} \int_{t_{0}}^{t_{1}} d t_{2} \phi_{c l}\left(t_{2}\right) \Phi_{3}\left(t_{1}, t_{2}\right)\right\rangle_{i . c .},
$$

where $\phi_{c l}$ is the Jacobi elliptical function (21) and $V_{3}$ is the full volume of the system (19).

Spatially homogeneous case of variations eq.(18) depends only on two times. Since we know analytical solution for $\phi_{c l}$ and the first variation $\Phi_{1}\left(t_{1}, t_{2}\right)$ (see [21]) it is clear that all three variations are periodic functions (with period of the classical solution (21)) multiplied by some power of $t_{1}$. It is easy to show that $k$-th variation grows as $t_{1}^{k}$ term at most.

The limits of integration over $t_{2}$ in NLO term of eq.(22) show that this term is subleading at small times $t_{1}$ and we can consider behavior of NLO term only at $t_{1}>>t_{0}$. At this limit we can neglect phase $C$ in eq.(21) and introduce new dimensionless variable $z=g \phi_{m} t$. It help us obtain dimensionless variations $f_{n}\left(z_{1}, z_{2}\right)$ as

$$
\phi_{c l}\left(t_{1}\right)=\phi_{m} f_{0}\left(z_{1}\right), \quad \Phi_{k}\left(t_{1}, t_{2}\right)=g^{-k} \phi_{m}^{1-2 k} f_{k}\left(z_{1}, z_{2}\right), \quad k=1,2,3 .
$$

Dimensionless case of eq.(22) has the form

$$
\langle\hat{\varphi}(t)\rangle_{t_{1}}^{L O+N L O}=\left\langle\phi_{m} f_{0}\left(z_{1}\right)+\frac{1}{4 g^{2} V_{3}^{2} \phi_{m}^{5}} \int_{z_{0}}^{z_{1}} d z_{2} f_{0}\left(z_{2}\right) f_{3}\left(z_{1}, z_{2}\right)\right\rangle_{i . c .} .
$$

Because of periodicity of $f_{k}\left(z_{1}, z_{2}\right)$ the integral over $z_{2}$ behave at late times $\left(z_{1}>>z_{0}\right)$ as

$$
\int_{z_{0}}^{z_{1}} d z_{2} f_{0}\left(z_{2}\right) f_{3}\left(z_{1}, z_{2}\right) \approx z_{1}^{3} \psi\left(z_{1}\right)
$$

where $\psi\left(z_{1}\right)$ is a periodic function with same period $T_{c l}$. Finally, we have

$$
\langle\hat{\varphi}(t)\rangle_{t_{1}}^{L O+N L O} \approx\left\langle\phi_{m}\left[f_{0}\left(g \phi_{m} t_{1}\right)+\frac{g t_{1}^{3}}{4 V_{3}^{2} \phi_{m}^{3}} \psi\left(g \phi_{m} t_{1}\right)\right]\right\rangle_{i . c .} .
$$

It seems that NLO term of eq.(26) is dominated at large times $t_{1}$ before averaging on initial conditions. Therefore the choice of the Wigner function play a crucial role and dynamics of the system strongly depends on the initial state. This feature is rather general for far-from-equilibrium systems.

As it is shown in [21] in case of gaussian choice for the Wigner function

$$
\begin{gathered}
f_{W}(\alpha, p)=\frac{1}{\pi \alpha_{0} p_{0}} e^{-\frac{(\alpha-A)^{2}}{\alpha_{0}^{2}}} e^{-\frac{p^{2}}{p_{0}^{2}},}, \\
\langle\quad\rangle_{i . c .} \equiv \int \frac{d p}{2 \pi} \int d \alpha f_{W}\left(t_{0}, \alpha, p\right),
\end{gathered}
$$

it is possible to perform the averaging over initial conditions analytically. Let us make a change of variables $(\alpha, p) \rightarrow\left(\phi_{\max }, C\right)$ (see $\left.(21)\right)$ :

$$
\begin{gathered}
\int \frac{d p}{2 \pi} \int d \alpha \rightarrow \int|J| d \phi_{\max } d C . \\
\left|J\left(\phi_{\max }\right)\right|=g \phi_{\max }^{2} .
\end{gathered}
$$


Analytical integration over $\phi_{\max }$ and $C$ is possible in the saddle point approximation, where

$$
f_{W}\left(\phi_{\max }, C, 0\right) \approx \frac{1}{\alpha_{0} p_{0} \pi} e^{-\frac{\left(\phi_{\max }-A\right)^{2}}{\alpha_{0}^{2}}-\frac{C^{2} A^{4} g^{2}}{p_{0}^{2}}} .
$$

Let us introduce Fourier transform as

$$
\begin{gathered}
c n\left(\frac{1}{2} ; g \phi_{\max } t+C\right)=\sum_{k=-\infty}^{\infty} u_{k} e^{\frac{2 \pi i k}{T}\left(g \phi_{\max } t+C\right)}, \\
u_{m}=\frac{1}{T} \int_{0}^{T} c n\left(\frac{1}{2} ; t\right) e^{-i m t \frac{2 \pi}{T}} d t \\
\psi_{m}=\frac{1}{T} \int_{0}^{T} \psi(t) e^{-i m t \frac{2 \pi}{T}}
\end{gathered}
$$

where $T$ is the period of the Jacobi elliptical function $c n\left(\frac{1}{2} ; t\right)$. Performing these calculations for the field expectation value we obtain

$$
\langle\hat{\varphi}(t)\rangle_{t_{1}}^{L O+N L O} \approx 2 A \sum_{k=0}^{\infty}\left(u_{k}+\frac{g t_{1}^{3}}{4 V_{3}^{2} A^{3}} \psi_{k}\right) e^{-\frac{\pi^{2} p_{0}^{2}}{g^{2} A^{4} T^{2}} k^{2}} e^{-\frac{\alpha_{2}^{2} \pi^{2} g^{2}}{T^{2}} k^{2} t_{1}^{2}} \cos \left(\frac{2 A g \pi k}{T} t_{1}\right) .
$$

The common time behavior of both LO and NLO terms is oscillation with the amplitude that fall in time according to the cosine and the exponent of the eq.(32). However NLO term additionally grows as $t_{1}^{3}$. The asymptotic behavior of both terms are identical because of term $\exp \left(-t_{1}^{2}\right)$, but early time evolution (usually most interesting part of the problem) fully depend on initial state.

Parameter $A$ in the Wigner function (27) denotes the measure of excitation of the initial field configurations. It means that starting points of all classical trajectories $\phi_{c l}\left(t_{0}\right) \equiv \alpha$ are grouped around some value $A$. In order to describe highly-excited fields at the initial state (that is what we want for heavy ion collisions for example) this parameter should be large $A>>1$. As one can see from (32) it means that NLO term is suppressed.

Similar analysis can be performed for the $T^{\mu v}$ components

$$
T^{\mu \nu}=\partial^{\mu} \varphi \partial^{v} \varphi-g^{\mu \nu}\left(\frac{1}{2} \partial_{\sigma} \varphi \partial^{\sigma} \varphi-\frac{g^{2}}{4} \varphi^{4}\right)
$$

At the classical level energy and pressure are:

$$
\varepsilon_{c l}=\frac{1}{2} \dot{\phi}_{c l}^{2}+\frac{g^{2}}{4} \phi_{c l}^{4}, p_{c l}=\frac{1}{2} \dot{\phi}_{c l}^{2}-\frac{g^{2}}{4} \phi_{c l}^{4} .
$$

At the LO level we have

$$
\begin{aligned}
& \varepsilon_{L O}\left(t_{1}\right)=\left\langle\frac{1}{2} \dot{\phi}_{c l}^{2}\left(t_{1}\right)+\frac{g^{2}}{4} \phi_{c l}^{4}\left(t_{1}\right)\right\rangle_{i . c .}, \\
& p_{L O}\left(t_{1}\right)=\left\langle\frac{1}{2} \dot{\phi}_{c l}^{2}\left(t_{1}\right)-\frac{g^{2}}{4} \phi_{c l}^{4}\left(t_{1}\right)\right\rangle_{i . c .} .
\end{aligned}
$$

These result had been obtained by [1]. However, one need to note that the NLO answers of [1] correspond to LO observables in our work. 
At the NLO level $\varepsilon_{N L O}=0$. It is conserved, but at the initial moment $\varepsilon_{N L O}\left(t_{0}\right)=0$ due to integration over $t_{2}$ in (3.2). For $p_{N L O}$ we have

$$
\langle\hat{p}(t)\rangle_{t_{1}}^{L O+N L O}=\left\langle g^{2} \phi_{m}^{4}\left[\pi_{L O}\left(g \phi_{m} t_{1}\right)+\frac{g t_{1}^{3}}{4 V_{3}^{2} \phi_{m}^{3}} \pi_{N L O}\left(g \phi_{m} t_{1}\right)\right]\right\rangle_{i . c .},
$$

where

$$
\pi_{L O}\left(z_{1}\right)=\frac{1}{2}\left(f_{0}^{\prime}\left(z_{1}\right)\right)^{2}-\frac{1}{4} f_{0}^{4}\left(z_{1}\right)
$$

and

$$
\begin{aligned}
\int_{z_{0}}^{z_{1}} d z_{2} f_{0}\left(z_{1}\right)\left[f_{0}^{\prime}\left(z_{1}\right) f_{3}^{\prime}\left(z_{1}, z_{2}\right)\right. & +3 f_{1}^{\prime}\left(z_{1}, z_{2}\right) f_{2}^{\prime}\left(z_{1}, z_{2}\right)- \\
-f_{0}^{3}\left(z_{1}\right) f_{3}\left(z_{1}, z_{2}\right)-9 f_{0}^{2}\left(z_{1}\right) f_{1}\left(z_{1}, z_{2}\right) f_{2}\left(z_{1}, z_{2}\right) & \left.\quad-6 f_{0}\left(z_{1}\right) f_{1}^{3}\left(z_{1}, z_{2}\right)\right] \approx z_{1}^{3} \pi_{N L O}\left(z_{1}\right) .
\end{aligned}
$$

Again $\pi_{L O}, \pi_{N L O}$ are periodic functions with period $T_{c l}$.

One can note the dimensionless combination

$$
c_{1}\left(g \phi_{m} t_{1}\right)+\frac{g t_{1}^{3}}{4 V_{3}^{2} \phi_{m}^{3}} c_{2}\left(g \phi_{m} t_{1}\right)
$$

which in both examples eq.(26) and eq.(37) is similar and corresponds to LO + NLO terms. As $\phi_{m}=\phi_{m}(\alpha, p)$ depends on initial conditions we can see that integration with the Wigner function which gives small amplitude $\phi_{m}$ (i.e. has small height and wide tails) results in case where NLO term became significant.

\section{Conclusions}

Let us summarize the main results presented in the talk.

- Systematic formalism for calculating corrections to the semiclassical approximation for correlators of highly excited quantum field in the framework of Keldysh technique is presented. It is shown that the results obtained in the leading semiclassical approximation reproduce those within the Classical Statistical Aproximation (CSA) thus establishing equivalence between the two approaches. An important feature of the CSA approximation is that it is generically not a small-coupling one.

- Generic expressions for leading corrections to the CSA approximation for the scalar field case were derived.

- Analytical expressions for the mean field, energy and pressure of the homogeneous scalar field were derived. The critical role of the character of initial conditions for applicability of the CSA approximation was discussed.

AR was supported by the Russian Science Foundation under grant No. 14-02-31748 


\section{References}

[1] K. Dusling, T. Epelbaum, F. Gelis, R. Venugopalan, Nucl.Phys. A850, 69-109 (2011)

[2] T. Epelbaum, F. Gelis, Nucl.Phys. A872, 210-244 (2011)

[3] K. Dusling, T. Epelbaum, F. Gelis, R. Venugopalan, Phys.Rev. D86, 085040 (2012)

[4] K. Fukushima, F. Gelis, L. McLerran, Nucl.Phys. A786, 107-130 (2007)

[5] J. Berges, K. Boguslavski, S. Schlichting, et al., J. High Energ. Phys. 1405, 54 (2014)

[6] A. Kurkela and Y. Zhu, Phys. Rev. Lett. 115, 182301 (2015)

[7] L. Keegan, A. Kurkela, A. Mazeliauskas and D. Teaney, arXiv:1605.04287 [hep-ph]

[8] A. Kurkela, arXiv:1601.03283 [hep-ph]

[9] D. T. Son, hep-ph/9601377.

[10] S. Y. Khlebnikov and I. I. Tkachev, Phys. Rev. Lett. 77, 219 (1996)

[11] D. Boyanovsky, Phys. Rev. D 92, 023527 (2015)

[12] E. T. Akhmedov, H. Godazgar and F. K. Popov, Phys. Rev. D 93, 024029 (2016)

[13] E. T. Akhmedov and F. K. Popov, JHEP 1509, 085 (2015)

[14] E. T. Akhmedov, N. Astrakhantsev and F. K. Popov, JHEP 1409, 071 (2014)

[15] F. Gelis and N. Tanji, Prog. Part. Nucl. Phys. 87, 1 (2016)

[16] J. Berges and T. Gasenzer, Phys. Rev. A 76, 033604 (2007)

[17] Kean Loon Lee, Nick P. Proukakis, arXiv:1607.06939 [cond-mat.quant-gas]

[18] L.V. Keldysh, Zh.Eksp.Teor.Fiz. 47 (1964) 1515-1527; Sov.Phys.JETP 20 (1965) 1018

[19] J. Berges, AIP Conf.Proc. 739,3-62 (2005)

[20] J. Berges, e-Print: arXiv:1503.02907

[21] A. V. Leonidov and A. A. Radovskaya, JETP Lett. 101, 215 (2015)

[22] H. Lee, M.O. Scully, Journal of Chemical Physics 73, 2238 (1980)

[23] L.Bonnet, Journal of Chemical Physics 139, 114108 (2013)

[24] S. Jeon, Annals Phys. 340 (2014) 119-170 